\title{
Differential expression of microRNAs may regulate pollen development in Brassica oleracea
}

\author{
J.H. Song*, J. Yang*, F. Pan and B. Jin \\ Department of Vegetable, College of Horticulture, Anhui Agricultural University, \\ Hefei, China \\ *These authors contributed equally to this study. \\ Corresponding author: J.H. Song \\ E-mail: jianghua_80@126.com
}

Genet. Mol. Res. 14 (4): 15024-15034 (2015)

Received May 4, 2015

Accepted July 6, 2015

Published November 24, 2015

DOI http://dx.doi.org/10.4238/2015.November.24.10

\begin{abstract}
MicroRNAs (miRNAs) are a class of non-coding endogenous negative regulators that regulate gene expression at both the transcriptional and post-transcriptional levels. However, little is known about the expression characteristics of miRNAs during pollen development in Brassica oleracea. In this study, five known and three novel miRNAs were identified and their expression patterns were compared in the flower buds of $B$. oleracea using stem-loop reverse transcription polymerase chain reaction (RT-PCR) and quantitative real-time PCR. The results revealed that the eight miRNAs were constantly expressed during pollen development but exhibited different expression patterns during the five developmental stages of the flower buds between the cytoplasmic male sterile (CMS) line and its fertile maintainer. The highest miRNA expression levels occurred at the uninucleate microspore stage in the fertile line $B 001-12 B$ and at the bicellular pollen stage in the CMS line Bo01-12A. Potential target genes for the miRNAs were predicted and analyzed, and suggested that miRNAs are involved in the regulation of target genes related to pollen development.
\end{abstract}


The results of this study further our understanding of the regulatory role of miRNAs in pollen development.

Key words: miRNA; Expression pattern; Flower bud; Male sterility

\section{INTRODUCTION}

MicroRNAs (miRNAs) are 21-24-nucleotide regulatory non-coding RNAs that play critical roles in transcriptional and post-transcriptional gene regulation in animal and plant life processes. Plant miRNAs are essential for plant growth and development, including organ morphogenesis, phase change, fruit development, and defense against biotic and abiotic stress (Chen, 2009; Kumar, 2014). Hundreds of plant miRNAs and their targets that regulate plant development have been identified by experimental or computational approaches (Alves et al., 2009; Han et al., 2014; Liu et al., 2014b; Zhang and Wang, 2015). Most of them are deeply conserved in plants, but many have been found to be tissue-specific, stage-specific, or genotype-dependent (Oh et al., 2008; Zhang et al., 2012; Sun et al., 2014). Some stage-specific miRNAs have been isolated and functionally characterized with respect to their roles in the reproductive biology of Arabidopsis, soybean, and other plant species (Zhan and Lukens, 2010; Shamimuzzaman and Vodkin, 2012; Wang et al., 2014). Differences in stage-specific miRNA expression patterns provide information on their possible regulatory functions.

Pollen development is a complex process that is crucial for sexual reproduction in higher plants. Significant progress has been made in pollen research by employing a variety of resources and novel techniques (Jiang et al., 2014), which have provided important information regarding the molecular mechanism of pollen development and the control of crop fertility. Recently, it has been demonstrated that miRNAs are differentially expressed during the various periods of pollen development, and play specific roles in regulatory function in model plants (Le Trionnaire and Twell, 2010; Wei et al., 2011). For example, miRNAs have been shown to function in mature Arabidopsis pollen, together with some of the most important genes involved in the miRNA silencing pathway, such as DCL1, AGO1, and RDR6 (Grant-Downton et al., 2009a). However, the diversity of miRNAs and their potential roles in pollen development in Brassica oleracea have rarely been investigated.

$B$. oleracea is one of the most important commercial vegetable crops, and has been eaten by humans for thousands years. Recently, $10 \mathrm{~B}$. oleracea miRNAs were deposited in the publicly available miRNA database (miRBase, release 21). We previously screened preferentially expressed miRNA sequences in $B$. oleracea flower buds using high-throughput sequencing and bioinformatic analysis (Yang and Song, 2014). In the present study, the expression levels of five known and three novel miRNAs during pollen development were identified and validated using stem-loop reverse transcription polymerase chain reaction (RT-PCR) and quantitative real-time PCR (qPCR). The characteristics of their target genes were then analyzed in order to understand the possible functions of these miRNAs during pollen development in B. oleracea.

\section{MATERIAL AND METHODS}

\section{Plant materials}

Cytoplasmic male sterile (CMS) line $B$. oleracea 'Bo01-12A' and its fertile maintainer line 'Bo01-12B' were grown in a greenhouse under standard conditions. Flower buds were classified into 
five developmental stages, depending on their longitudinal diameters and cytological observations (Kang et al., 2014). The flower buds were collected, frozen immediately in liquid nitrogen, and stored at $-80^{\circ} \mathrm{C}$ for subsequent total RNA isolation. The developmental stages of the flower buds were as follows: $\mathrm{S} 1(0-1 \mathrm{~mm})$, mostly sporogenous cell to pollen mother cell stage; S2 (1-2 $\mathrm{mm})$, mostly tetrad stage; S3 (2-3.5 mm), mostly uninucleate microspore stage; S4 (3.5-5 mm), mostly bicellular pollen stage; S5 (>5 mm), mostly tricellular pollen stage to mature pollen grain stage.

\section{miRNA primer design}

The mature miRNA sequences were acquired by deep sequencing. In the present study, eight miRNAs were selected because of their significantly preferential expression in the flower buds, and included five known miRNAs (bol-miR157a, bol-miR171a, bol-miR172, bol-miR824, and bol-miR398a-3p) and three novel miRNAs (bol-miR0023, bol-miR0101, and bol-miR0142). Primers for reverse transcription and qPCR are listed in Table 1. Stem-loop primers were designed according to the method described by Kramer (2011). The reverse primer for miRNA qPCR is a universal one, but the forward primers were designed according to the miRNA sequences.

\section{Total RNA isolation and cDNA synthesis}

Total RNA was extracted from each sample using TRIzol reagent (Takara, Japan) according to the manufacturer instructions. RNA concentration and purity were checked using a NanoDrop ${ }^{\text {TM }} 1000$ spectrophotometer (Thermo Scientific). First-strand cDNA was synthesized using a PrimeScript ${ }^{T M}$ RT reagent kit (Takara) according to the manufacturer instructions. To increase reverse-transcription efficiency, a pulsed $\mathrm{RT}$ reaction was used $\left(42^{\circ} \mathrm{C}\right.$ for $15 \mathrm{~min}$, followed by a final reverse-transcriptase inactivation at $85^{\circ} \mathrm{C}$ for $5 \mathrm{~s}$ and reservation at $4^{\circ} \mathrm{C}$ ).

\section{Qualitative analysis}

Stem-loop RT-PCR was conducted for qualitative expression analysis. As an internal control and to exclude genomic contamination, glyceraldehyde 3-phosphate dehydrogenase (GAPDH) was amplified from the same cDNA samples. Stem-loop RT-PCR was conducted using an Applied Biosystems 9700 Thermal Cycler. The $25-\mu \mathrm{L}$ reaction mixture consisted of $2.5 \mu \mathrm{L} 10 \mathrm{X}$ PCR buffer, $0.5 \mu \mathrm{L} 0.25 \mathrm{mM}$ dNTP mixture, $0.5 \mu \mathrm{L}$ forward primer, $0.5 \mu \mathrm{L}$ reverse primer, $0.2 \mu \mathrm{L}$ Taq DNA polymerase (Takara), and $0.8 \mu \mathrm{L}$ template cDNA. Amplifications were performed with the following cycle conditions: $94^{\circ} \mathrm{C}$ for $3 \mathrm{~min}, 94^{\circ} \mathrm{C}$ for $30 \mathrm{~s}, 63^{\circ} \mathrm{C}$ for $30 \mathrm{~s}, 30$ cycles at $72^{\circ} \mathrm{C} \mathrm{for}$ $30 \mathrm{~s}$, and $72^{\circ} \mathrm{C}$ for $1 \mathrm{~min}$. The PCR products were checked and visualized by $2 \%$ agarose gel electrophoresis with ethidium bromide staining.

\section{qPCR assay}

The expression levels of the checked miRNAs were examined by qPCR. Total RNA was prepared from flower buds at different developmental stages and converted to cDNA using a PrimeScript $^{\text {TM }}$ RT reagent kit (Takara). Three replicates were included. The qPCR was performed using a SYBR ${ }^{\circledR}$ Premix Ex Taq II kit (Takara) on a CFX96 ${ }^{\text {TM }}$ Real-Time System (Bio-Rad, USA). The 25- $\mu \mathrm{L}$ PCR mixture contained $2.0 \mu \mathrm{L}$ cDNA templates, $12.5 \mu \mathrm{L} 2 X$ SYBR ${ }^{\circledR}$ Premix Ex Taq II, $1.0 \mu \mathrm{L}$ forward primer, $1.0 \mu \mathrm{L}$ reverse primer, and $8.5 \mu \mathrm{L} \mathrm{ddH_{2 }} \mathrm{O}$. The reaction mixtures were incubated at 
$95^{\circ} \mathrm{C}$ for $30 \mathrm{~s}$, followed by 40 cycles at $95^{\circ} \mathrm{C}$ for $5 \mathrm{~s}$ and $60^{\circ} \mathrm{C}$ for $30 \mathrm{~s}$. GAPDH was the only internal control. Results were viewed using CFX Manager software (Bio-Rad) and exported to Microsoft Excel. The $2^{-\Delta \Delta \mathrm{Ct}}$ method was used to analyze the relative transcript levels (Livak and Schmittgen, 2001).

\section{Prediction of miRNA target genes}

The targets of the identified miRNAs were predicted using the online software psRNATarget (http://plantgrn.noble.org/psRNATarget/) with the default parameters set (Dai and Zhao, 2011). Because B. oleracea genome information was unavailable, we used the Arabidopsis Gene Index for the target search. A BLASTx (http://blast.ncbi.nlm.nih.gov/blast/Blast. cgi?PROGRAM=blastx\&PAGE_TYPE=BlastSearch\&LINK_LOC=blasthome) search against the National Center for Biotechnology Information protein database (http://www.ncbi.nlm.nih.gov/ protein/) was performed to predict the functions of the potential targets.

\section{RESULTS}

\section{Identification of miRNAs during $B$. oleracea pollen development}

Using the total RNA from flower buds at each developmental stage of the two types of plant as a template, a PCR was conducted with the primers listed in Table 1. The resulting amplicons were of the predicted length ( $80 \mathrm{bp}$; Figure 1). All eight miRNAs, including bol-miR157a, 171a, $172,824,398 a-3 p$, and novel bol-miR0023, 0101, and 0142 were detected at all five developmental stages in both the CMS line 'Bo01-12A' and its fertile maintainer line 'Bo01-12B'. This indicated that these miRNAs were constantly expressed during pollen development in $B$. oleracea.

Table 1. miRNAs and primer sequences used for reverse transcription polymerase chain reaction and quantitative polymerase chain reaction analysis in Brassica oleracea.

\begin{tabular}{llll}
\hline miRNA & Mature sequence & Stem-loop primer & Forward primer \\
\hline bol-miR157a & UUGACAGAAGA- & GTCGTATCCAGTGCAGGGTCCGAGG & ACACTCCAGCTGGG- \\
& UAGAGAGCAC & TATTCGCACTGGATACGACGTGCTC & TTGACAGAAGATAG \\
bol-miR171a & UUGAGCCGUG- & GTCGTATCCAGTGCAGGTCCGAGG & AGGCTCAGCTGTTG- \\
& CAAUAUCACG & TATTCGCACTGGATACGACCGTGAT & AGCCGTGCCAAT \\
bol-miR172 & AGAACUUGAU- & GTCGTATCCAGTGCAGGGTCCGGG & CGCCGCCAGCTGG- \\
& GAUGCUGCAU & TATTCGCACTGGATACGACATGCAG & AGATCTTGATGATG \\
bol-miR398a-3p & UGUGUUCUCAG- & GTCGTATCCAGTGCAGGGTCCGAGG & GTGTTCTCAGGTCA \\
& GUCACCCCUU & TATTCGCACTGGATACGACAAGGGG & ACCGTCCAGCTGGT- \\
bol-miR824 & UAGACCAUUUG- & GTCGTATCCAGTGCAGGGTCCGGG & AGACCATTTGGAG \\
& UGAGAAGGGA & TATTCGCACTGGATACGACTCCCTT & GTCTCCAGCTGGGC- \\
bol-miR0023 & GCAAGTTGACT- & GTCGTATCCAGTGCAGGGTCCGAGG & AAGTTGACTTTGG \\
& TTGGCTCTGT & TATTCGCACTGGATACGACACAGAG & TCCAGCTGGCTTG- \\
bol-miR0101 & CTTGACTAGGA- & GTCGTATCCAGTGCAGGGTCCGAGG & ACTAGGACGGTCTG \\
bol-miR0142 & GTCTGAGGCTT & TATTCGCACTGGATACGACAAGCCT & AGGCTCAGCTGCC- \\
& CCTTCTCATCG- & GTCGTATCCAGTGCAGGGTCCGAGG & TTCTCATCGATGG \\
\hline
\end{tabular}

Universal reverse primer, GGTCCGAGGTATTCGCACTGGATAC. All sequences are written in 5'-3'.

\section{miRNA expression patterns at different developmental stages}

To further characterize and validate the possible regulation of miRNAs at different stages of pollen development, qPCR was performed using a standard SYBR ${ }^{\circledR}$ PCR protocol with the identified miRNAs. The expression levels of the five known and three novel miRNAs at five critical 
developmental stages were analyzed between 'Bo01-12A' and 'Bo01-12B', and the temporal expression profiles of bol-miR157a, 171a, 172, 824, and 398a-3p, and novel bol-miR0023, 0101, and 0142 are shown in Figure 2.

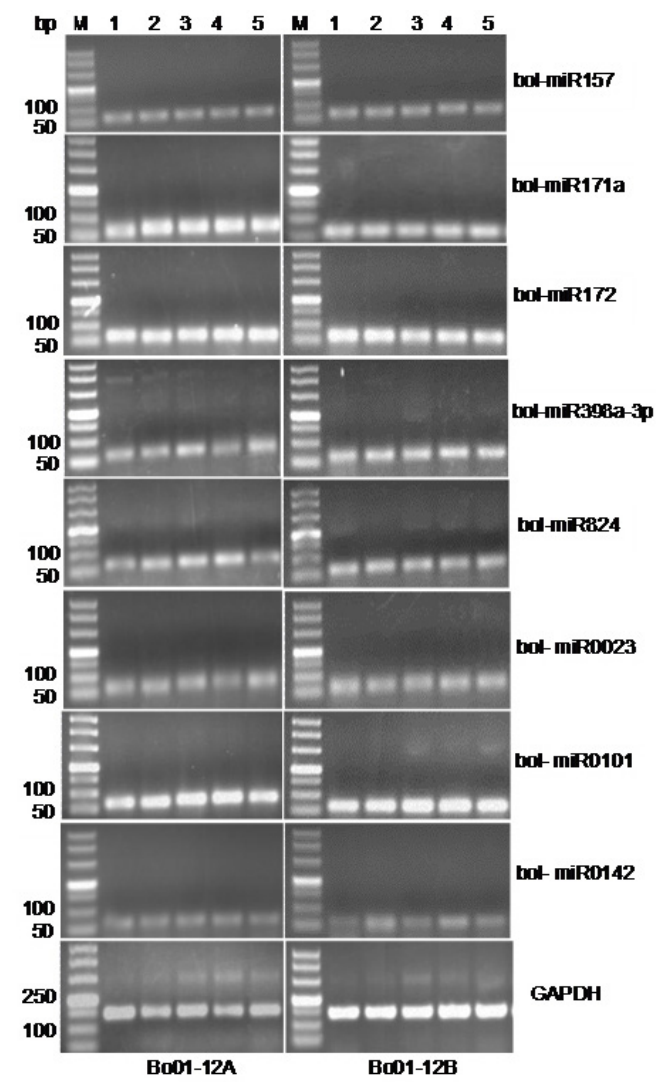

Figure 1. Reverse transcription polymerase chain reaction analysis of miRNA expression at different stages of pollen development in Brassica oleracea. Lane 1, sporogenous cell to pollen mother cell stage; lane 2, tetrad stage; lane 3 , uninucleate microspore stage; lane 4, bicellular pollen stage; lane 5, tricellular pollen stage to mature pollen grain stage; Bo01-12A, cytoplasmic male sterile line; Bo01-12B, fertile maintainer line; lane M, 500-bp DNA Ladder.

bol-miR157a expression in the CMS line 'Bo01-12A' increased from stages 1 to 4 before significantly decreasing at stage 5 ; however, in 'Bo01-12B', it slightly decreased at first, then increased from stages 1 to 3 , before decreasing again at stage 4 (Figure 2A). There were no obvious trends in bol-miR171a expression in either line; the highest bol-miR171a expression level was at stage 4 in 'Bo01-12A' and at stage 3 in 'Bo01-12B' (Figure 2B). bol-miR172 expression levels were similar from stages 1 to 3 in both lines. However, it increased rapidly at stage 4 and stabilized at stage 5 in the CMS line 'Bo01-12A', whereas it decreased sharply at stage 4 and stabilized at stage 5 in the fertile line 'Bo01-12B' (Figure 2C). The expression levels of bol-miR398a-3p were lower in 'Bo01-12A' than in 'Bo01-12B' at stages 1 and 3, but higher in the CMS line at stages 2,4 , and 5 , with a significant difference at stages 4 and 5 (Figure 2D). During stages 1 to 3 , the expression levels of bol-miR824 in 'Bo01-12B' were always higher than those in 'Bo01-12A', and the same expression trends were maintained in both lines; subsequently, they were higher in the 
CMS line than in the fertile line at stages 4 and 5, and the same expression levels were maintained in each line (Figure 2E).

The expression levels of novel bol-miR0023, 0101, and 0142 were higher in the CMS line than in the fertile maintainer line, except at stage 3 (Figure 2F-H). The highest expression levels of the eight miRNAs all occurred at stage 4 in the CMS line and at stage 3 in the fertile maintainer line.
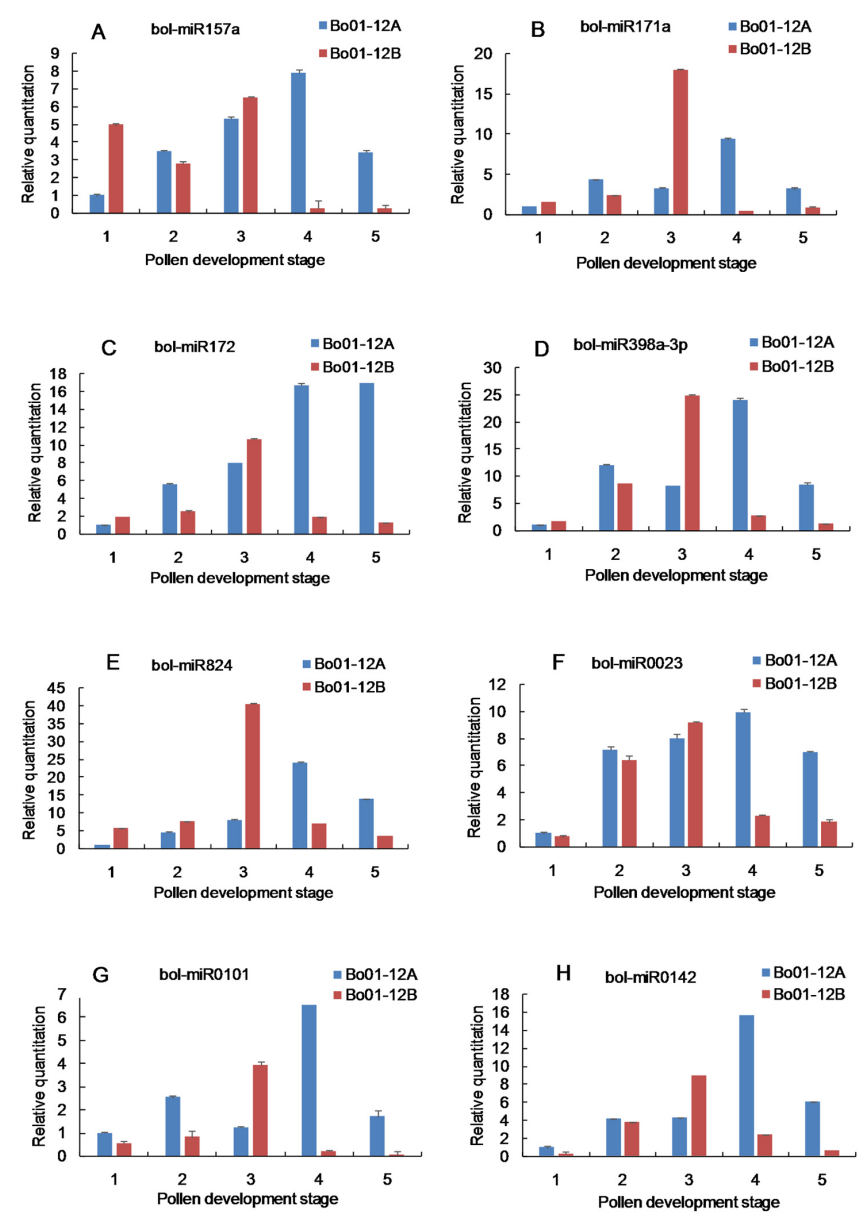

Figure 2. Quantitative polymerase chain reaction analysis of miRNA expression at different stages of pollen development in cytoplasmic male sterile and fertile maintainer lines of Brassica oleracea.

\section{Potential targets of the miRNAs during pollen development}

To understand the functions of the eight miRNAs, their potential target sites were predicted using psRNATarget: 92 target genes were predicted (Table 2). Although information regarding the target proteins was unavailable for the partial miRNAs, target protein identity was available for most of the known miRNAs, except for bol-miR398a-3p. The targets of the five known miRNAs encode special proteins that are associated with the stress response, transcription factors regulating gene expression, and enzymes relevant to metabolic and signaling pathways that are involved in pollen development. 


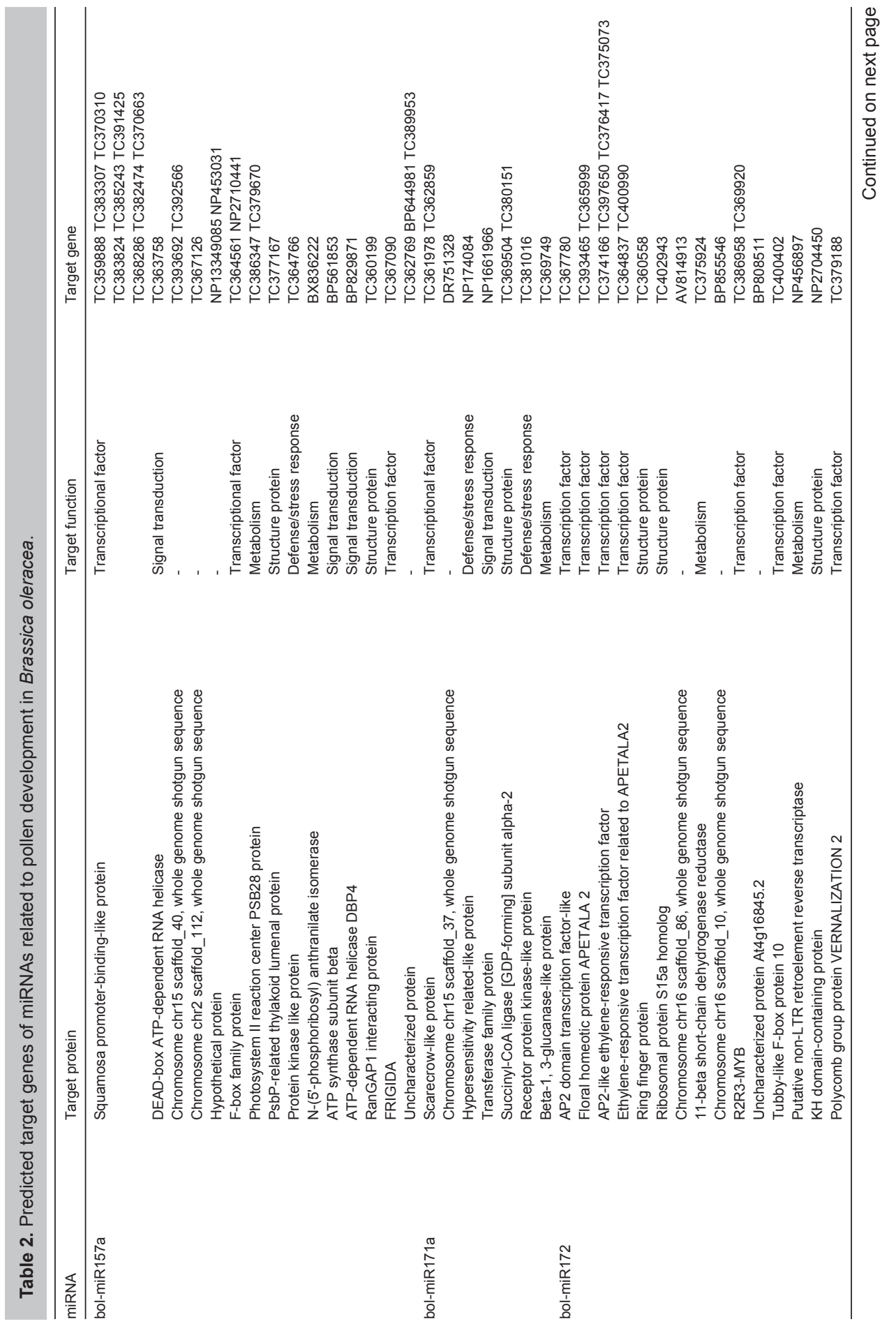




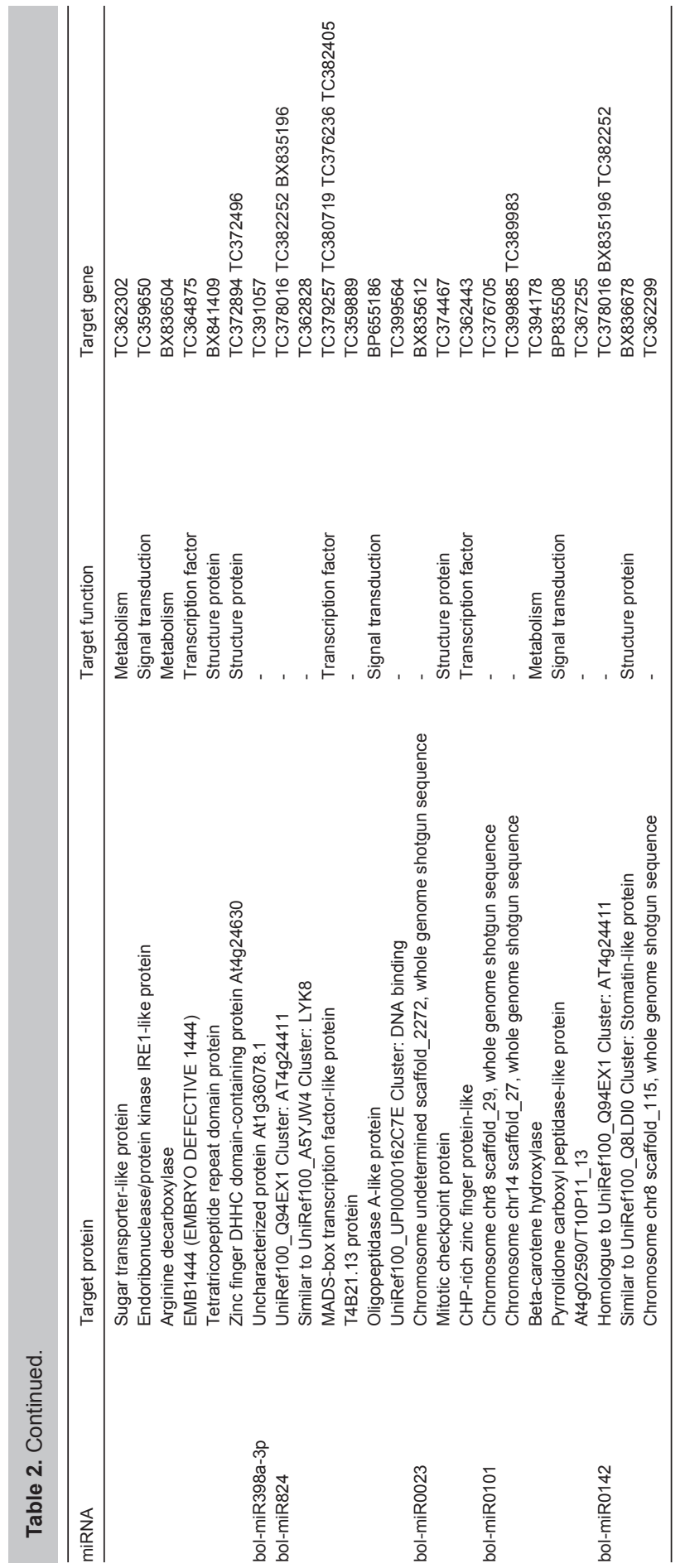




\section{DISCUSSION}

The expression information of any molecules, including miRNAs, at specific developmental stages as well as in specific tissues is of great significance for understanding their functions (Feng et al., 2014). Stem-loop RT-PCR is a method that exhibits high sensitivity and specificity for detecting miRNAs in animals and plants (Chen et al., 2005; Varkonyi-Gasic et al., 2007). In this study, qualitative RT-PCR with a stem-loop primer was performed to detect the presence of three novel and five known miRNAs during pollen development in CMS and fertile lines. All eight miRNAs were detectable at all five stages of pollen development. Previous reports have indicated that many miRNAs exist in pollen and may regulate pollen and anther development (Chambers and Shuai, 2009). For example, miR157, miR171, miR172, and miR824 were identified in mature Arabidopsis pollen using 454 sequencing and a miRCURY array (Chambers and Shuai, 2009; Grant-Downton et al., 2009b). miR171a and miR398 have been detected in male loblolly pine (Pinus taeda) gametophytes and rice pollen, respectively, by microarray analysis (Wei et al., 2011; Quinn et al., 2014). miR172b expression has been detected in the flower buds of Brassica campestris (Jiang et al., 2013). Therefore, the results of this and previous studies suggest that miRNAs are constantly expressed during pollen development.

A growing body of evidence suggests that miRNAs are an essential regulatory component of plants (Liu et al., 2014a). In order to quantify changes in miRNA expression during pollen development, we performed a qPCR-based assay that allowed us to detect miRNAs at low abundance and acquire precise data on relative miRNA expression levels. The eight miRNAs tested exhibited differential expression patterns during the five typical stages of pollen development between CMS and fertile lines. Recent studies have indicated that small-RNA pathways interfere with microgametogenesis, from mononuclear microspores to the tricellular pollen state, in Arabidopsis (Honys and Twell, 2004; Pina et al., 2005). Interestingly, we found that a transition in miRNA expression levels occurred at the uninucleate and bicellular microspore stages in fertile and CMS lines, respectively, of $B$. oleracea. Furthermore, each of the tested miRNAs tended to be sharply downregulated at the later stages of microspore development in the fertile line. Similarly, previous research has shown that most miRNAs are expressed at a very low abundance in mature Arabidopsis pollen (Chambers and Shuai, 2009). Therefore, the differential miRNA expression between the CMS and fertile lines might contribute to regulating pollen development and male sterility.

The identification of miRNA target genes is an important step in understanding miRNA regulation during plant development. To elucidate the functions of the miRNAs that were differentially expressed during pollen development in $B$. oleracea, we predicted their putative target genes. Since most plant miRNAs exhibit perfect or near-perfect complementarities with their target mRNAs, and B. oleracea and Arabidopsis have nucleotide sequence identities of 80-90\% (Amagai et al., 2003), potential target genes could easily be predicted using computational and homolog search methods. Many of the known targets encode transcription factors, which play an important role in plant growth and development (Jones-Rhoades and Bartel, 2004; Earley et al., 2010). For example, bol-miR157 targets the transcription factor SBP, which might participate in gametophyte development (Xing et al., 2010). bol-miR172 targets AP2-like transcription factors, which have been implicated in the regulation of flowering time and floral organ identity in Arabidopsis, maize, tobacco, and the opium poppy (Aukerman and Sakai, 2003; Chen, 2004; Frazier et al., 2010; Unver et al., 2010). Many miRNAs are evolutionarily conserved across a variety of plant species, and function in the regulatory control of fundamentally important biological processes (Xie et al., 2010). It is well known that MYB transcription factors are a superfamily of proteins that play regulatory roles 
in developmental processes and defense responses (Chen et al., 2006). The results of the present study suggest that MYB proteins may be one of the targets of bol-miR172a, and that miRNAs may be involved in the important functional regulation of pollen development in $B$. oleracea.

In summary, this study identified differential miRNA expression between CMS and fertile lines of $B$. oleracea during pollen development. The potential target gene predictions for the miRNAs further demonstrated that the expressional patterns of these miRNAs could modulate pollen developmental processes. These results strongly suggest that these miRNAs are constantly expressed and temporally regulate non-coding small RNA that is involved in $B$. oleracea pollen development. Further studies are needed to elucidate the specific roles of miRNAs in pollen development, in order to provide a better understanding of the regulatory mechanisms of pollen development at the miRNA level.

\section{Conflicts of interest}

The authors declare no conflict of interest.

\section{ACKNOWLEDGMENTS}

Research supported by the National Natural Science Foundation of China (\#31272170 and \#30800750) and the Natural Science Foundation of Anhui Province (\#1508085MC51).

\section{REFERENCES}

Alves LJ, Niemeier S, Hauenschild A, Rehmsmeier M, et al. (2009). Comprehensive prediction of novel microRNA targets in Arabidopsis thaliana. Nucleic Acids Res. 37: 4010-4021.

Amagai M, Ariizumi T, Endo M, Hatakeyama K, et al. (2003). Identification of anther-specific genes in a cruciferous model plant, Arabidopsis thaliana, by using a combination of Arabidopsis macroarray and mRNA derived from Brassica oleracea. Sex Plant Reprod. 15: 213-220.

Aukerman MJ and Sakai $\mathrm{H}$ (2003). Regulation of flowering time and floral organ identity by a microRNA and its APETALA2-like target genes. Plant Cell 15: 2730-2741.

Chambers C and Shuai B (2009). Profiling microRNA expression in Arabidopsis pollen using microRNA array and real-time PCR. BMC Plant Biol. 9: 87-96.

Chen CF, Ridzon DA, Broomer AJ, Zhou ZH, et al. (2005). Real-time quantification of micro-RNAs by stem-loop RT-PCR. Nucleic Acids Res. 33: 179-187.

Chen XM (2004). A microRNA as a translational repressor of APETALA2 in Arabidopsis flower development. Science 303 : 2022-2025.

Chen XM (2009). Small RNAs and their roles in plant development. Annu. Rev. Cell Dev. Biol. 25: 21-44.

Chen YH, Yang XY, He K, Liu MH, et al. (2006). The MYB transcription factor superfamily of Arabidopsis: expression analysis and phylogenetic comparison with the rice MYB family. Plant Mol. Biol. 60: 107-124.

Dai XB and Zhao PX (2011). psRNATarget: a plant small RNA target analysis server. Nucleic Acids Res. 39: W155-W159.

Earley K, Smith M, Weber R, Gregory B, et al. (2010). An endogenous F-box protein regulates ARGONAUTE1 in Arabidopsis thaliana. Silence 1: 15.

Feng YP, Chen JF, Huang P, Wang X, et al. (2014). Expression analysis of differentially expressed miRNAs in male and female chicken embryos. Genet. Mol. Res. 13: 3060-3068.

Frazier TP, Xie FL, Freistaedter A, Burklew CE, et al. (2010). Identification and characterization of microRNAs and their target genes in tobacco (Nicotiana tabacum). Planta 232: 1289-1308.

Grant-Downton R, Hafidh S, Twell D and Dickinson HG (2009a). Small RNA pathways are present and functional in the angiosperm male gametophyte. Mol. Plant 2: 500-512.

Grant-Downton R, Le Trionnaire G, Schmid R, Rodriguez-Enriquez J, et al. (2009b). MicroRNA and tasiRNA diversity in mature pollen of Arabidopsis thaliana. BMC Genomics 10: 643-658.

Han R, Jian C, Lv JY, Yan Y, et al. (2014). Identification and characterization of microRNAs in the flag leaf and developing seed of wheat (Triticum aestivum L.). BMC Genomics 15: 289. 
Honys D and Twell D (2004). Transcriptome analysis of haploid male gametophyte development in Arabidopsis. Genome Biol. 5: R85.

Jiang JX, Jiang JJ, Yang YF and Cao JS (2013). Identification of microRNAs potentially involved in male sterility of Brassica campestris ssp. chinensis using microRNA array and quantitative RT-PCR assays. Cell Mol. Biol. Lett. 18: 416-432.

Jiang JX, Lv ML, Liang Y, Ma ZM, et al. (2014). Identification of novel and conserved miRNAs involved in pollen development in Brassica campestris ssp. chinensis by high-throughput sequencing and degradome analysis. BMC Genomics 15: $146-158$.

Jones-Rhoades MW and Bartel DP (2004). Computational identification of plant microRNAs and their targets, including a stress-induced miRNA. Mol. Cell 14: 787-799.

Kang JE, Guo YY, Chen YJ, Li HL, et al. (2014). Upregulation of the AT-hook DNA binding gene BoMF2 in OguCMS anthers of Brassica oleracea suggests that it encodes a transcriptional regulatory factor for anther development. Mol. Biol. Rep. 41: 2005-2014.

Kramer MF (2011). Stem-loop RT-qPCR for miRNAs. Curr. Protoc. Mol. Biol. 15: 10.

Kumar R (2014). Role of microRNAs in biotic and abiotic stress responses in crop plants. Appl. Biochem. Biotechnol. 174: 93-115.

Le Trionnaire G and Twell D (2010). Small RNAs in angiosperm gametophytes: from epigenetics to gamete development. Gene Dev. 24: 1081-1085.

Liu N, Wu S, Van Houten J, Wang Y, et al. (2014a). Downregulation of AUXIN RESPONSE FACTORS 6 and 8 by microRNA 167 leads to floral development defects and female sterility in tomato. J. Exp. Bot. 65: 2507-2520.

Liu WN, Yu WN, Hou LY, Wang XY, et al. (2014b). Analysis of miRNAs and their targets during adventitious shoot organogenesis of Acacia crassicarpa. Plos One 9: e93438.

Livak KJ and Schmittgen TD (2001). Analysis of relative gene expression data using real-time quantitative PCR and the 2(T) (-Delta Delta C) method. Methods 25: 402-408.

Oh TJ, Wartell RM, Cairney J and Pullman GS (2008). Evidence for stage-specific modulation of specific microRNAs (miRNAs) and miRNA processing components in zygotic embryo and female gametophyte of loblolly pine (Pinus taeda). New Phytol. 179: 67-80.

Pina C, Pinto F, Feijo JA and Becker JD (2005). Gene family analysis of the Arabidopsis pollen transcriptome reveals biological implications for cell growth, division control and gene expression regulation. Plant Physiol. 138: 744-756.

Quinn CR, Iriyama R and Fernando DD (2014). Expression patterns of conserved microRNAs in the male gametophyte of loblolly pine (Pinus taeda). Plant Reprod. 27: 69-78.

Shamimuzzaman M and Vodkin L (2012). Identification of soybean seed developmental stage-specific and tissue-specific miRNA targets by degradome sequencing. BMC Genomics 13: 310.

Sun RR, Wang QL, Ma J, He QL, et al. (2014). Differentiated expression of microRNAs may regulate genotype-dependent traits in cotton. Gene 547: 233-238.

Unver T, Parmaksiz I and Dundar E (2010). Identification of conserved micro-RNAs and their target transcripts in opium poppy (Papaver somniferum L.). Plant Cell Rep. 29: 757-769.

Varkonyi-Gasic E, Wu RM, Wood M, Walton EF, et al. (2007). Protocol: a highly sensitive RT-PCR method for detection and quantification of microRNAs. Plant Methods 3: 12

Wang C, Leng XP, Zhang YY, Kayesh E, et al. (2014). Transcriptome-wide analysis of dynamic variations in regulation modes of grapevine microRNAs on their target genes during grapevine development. Plant Mol. Biol. 84: 269-285.

Wei LQ, Yan LF and Wang T (2011). Deep sequencing on genome-wide scale reveals the unique composition and expression patterns of microRNAs in developing pollen of Oryza sativa. Genome Biol. 12: R53.

Xie Z, Khanna K and Ruan S (2010). Expression of microRNAs and its regulation in plants. Semin. Cell Dev. Biol. 21: $790-797$.

Xing S, Salinas M, Hohmann S, Berndtgen R, et al. (2010). miR156-targeted and nontargeted SBP-Box transcription factors act in concert to secure male fertility in Arabidopsis. Plant Cell 22: 3935-3950.

Yang J and Song J H (2014). Research progress of miRNAs in plant pollen development. Chin. J. Cell Biol. 36: $1317-1322$.

Zhan SH and Lukens L (2010). Identification of novel miRNAs and miRNA dependent developmental shifts of gene expression in Arabidopsis thaliana. Plos One 5: e10157.

Zhang BH and Wang QL (2015). MicroRNA-based biotechnology for plant improvement. J. Cell Physiol. 230: 1-15.

Zhang JH, Zhang SG, Han SY, Wu T, et al. (2012). Genome-wide identification of microRNAs in larch and stage-specific modulation of 11 conserved microRNAs and their targets during somatic embryogenesis. Planta 236: 647-657. 